\title{
Pins and Needles: Minimally Invasive Office Techniques for Facial Rejuvenation
}

\author{
Corey S. Maas, M.D., ${ }^{1}$ and Sumit Bapna, M.D. ${ }^{1}$
}

\section{ABSTRACT}

The desire for minimally invasive facial rejuvenation has continued to increase from the perspective of both the patients and injectors. For successful rejuvenation, it is important to understand the anatomic changes of the aging face as well as the properties of available neuromodulators and soft tissue fillers. The injector should be knowledgeable of the advantages and disadvantages of each product. Patient selection, perhaps, plays the largest role in success, choosing patients that would truly benefit from and have reasonable expectations for minimally invasive techniques. Unsatisfactory outcomes can be limited by meticulous injection technique and well thought out treatment plans.

KEYWORDS: Injectables, fillers, botulinum toxin, facial rejuvenation, minimally invasive

The use of injectables has continued to increase in cosmetic surgery practices with the greater demands from patients for minimally invasive aesthetic improvement. ${ }^{1-8}$ This is secondary to not only the growing indications and availability of neuromodulators and dermal fillers (Table 1) but also the desire for rejuvenation from a wider patient population among varying ages and ethnicities. ${ }^{6}$ At present, Botox Cosmetic (Allergan, Inc., Irvine $\mathrm{CA}$ ) has the hold on the neuromodulator market, although there are recent developments in approval of competing drugs that will be discussed later. Currently available dermal fillers include porcine, bovine, and human collagens, hyaluronic acid (HA) preparations of animal or biosynthetic origin, poly-L-lactic acid products, polymethylmethacrylate, and calcium hydroxyapatite. ${ }^{3,7}$ Injection of soft tissue fillers and neuromodulators is an attractive office procedure for physicians because of the associated ease, cost, and minimal discomfort involved with treatment. It is important to select patients carefully and discuss expected benefits and limitations prior to treatment to avoid disappointment of patients

\footnotetext{
${ }^{1}$ The Maas Clinic, San Francisco, California.

Address for correspondence and reprint requests: Sumit Bapna, M.D., The Maas Clinic, 2400 Clay Street, San Francisco, CA 94115 (e-mail: drbapna@gmail.com).

Management of the Aging Face; Guest Editor, Anthony P.
}

because many aspects of facial rejuvenation still require surgical interventions. ${ }^{8}$

\section{NEUROMODULATORS}

Botulinum toxin, first used for the management of strabismus in the 1980s by Dr. Allen Scott in San Francisco, is now the workhorse of injectable agents for facial rejuvenation. In nature, the protein is found in seven different serotypes (A through G). The neuromodulator's biologic action occurs at the neurosynaptic cleft responsible for muscle contraction by cleaving proteins involved in the active transport of acetylcholine. Originally these proteins were described as toxins due to the illness botulism, which was associated with consuming large amounts of Clostridium botulinum-contaminated food. With the current widespread medical use, these proteins are better described as neuromodulators. Their desired action is selective weakening, relaxation, or paralysis of treated muscles or muscle groups. By selectively weakening specific hypertrophic muscle groups in
Sclafani, M.D., F.A.C.S.

Facial Plast Surg 2009;25:260-269. Copyright (C) 2009 by Thieme Medical Publishers, Inc., 333 Seventh Avenue, New York, NY 10001, USA. Tel: +1(212) 584-4662.

DOI 10.1055/s-0029-1242038. ISSN 0736-6825. 
Table 1 Injectables for Facial Rejuvenation

\begin{tabular}{lll}
\hline Neuromodulators & Target & Duration of Effect \\
\hline $\begin{array}{l}\text { Botulinum toxin type A } \\
\text { Botox }\end{array}$ & Muscle of facial expression & 3 to 4 months \\
Dysport & & \\
Purtox & & Duration of Correction \\
Botulinum toxin type B & & 3 months \\
Myobloc & Target & 4 months \\
Soft Tissue Fillers & Facial lines and folds & 3 to 4 months \\
Bovine collagen (Zyderm/Zyplast) & Deep lines and folds & 12 months \\
Avian hyaluronic acid (Hylaform) & Fine lines, lip border & 6 months \\
Human collagen (Cosmoderm) & Deep lines and folds & 12 months \\
Porcine collagen (Evolence) & & 9 months \\
Synthesized hyaluronic acids & Facial lines and folds, lip augmentation, tear trough \\
Restylane & Deformity & 3 to 4 months \\
Juvederm & Deeper lines and folds & 12 months \\
Perlane & Fine lines & Permanent \\
Prevelle & Deep lines and folds, facial volume enhancement & Permanent \\
Calcium hydroxyapatite particles (Radiesse) & Lines and folds, volume enhancement & \\
Silicone (Silikon 1000) & Facial volume enhancement &
\end{tabular}

the face and neck, unwanted lines and socially undesirable facial expressions can be suppressed or even eliminated. ${ }^{7,9,10}$

Both and $\mathrm{A}$ and $\mathrm{B}$ serotypes have been shown to have effectiveness for cosmetic applications. The B serotype, available as Myobloc (Myobloc Solstice Neurosciences, San Francisco, CA), has limited benefits under its current formulation secondary to shorter duration of action compared with that of the type A neuromodulator. ${ }^{11}$ It is also associated with more pain during injection, however it may have a faster onset of action.

The A serotype has longer duration of effect (90 to 120 days) and less discomfort with injection. Around the world, several brands of botulinum-A are available, whereas in the United States, only Botox (BTX; Allergan, Inc.) is commercially available, which has demonstrated a safety and efficacy record for more than 15 years and has set industry standards. Another brand, Dysport (Inamed, Inc., Santa Barbara, CA), available in Europe, Asia, and South America, recently received U.S. Food and Drug Administration (FDA) approval for cosmetic and functional applications and is soon to be released. The manufacturer reports a shorter onset of duration than that of BTX, however this is difficult to quantify because Allergan did not capture this data point in their clinical trials. The dosing for Dysport is 2.5 to 4 times the unit dose of BTX for equal effectiveness. ${ }^{12}$ Purtox (Mentor Corp., Santa Barbara, $\mathrm{CA}$ ), another botulinum-A, is nearing FDA approval.

The optimal use of BTX requires an intimate knowledge of the facial muscular anatomy and the functional interrelationships of the muscle actions. Many techniques and surface point injections have been shown to be successful related to the diffusion effects and relative safety of BTX without reducing the product's overall satisfactory clinical results. However, dosages for a given anatomic area can be reduced by precise localization into the targeted muscle or muscle groups. ${ }^{7}$

\section{NEUROMODULATOR APPLICATIONS}

Rejuvenation of the face and neck is achieved using BTX to target specific muscles and muscle groups. For ease of discussion of the anatomy and injection techniques, we will separate these into three distinct areas: upper face, lower face, and neck. Patients should be informed of the possible side effects and complications of each specific area treated. Injection of BTX does not involve any downtime, and localized erythema and subcutaneous edema is very transient. The injection techniques described later use a dilution of 50 units per milliliter using an insulin syringe with 31 -gauge needle.

In the aging upper face, four main problem areas exist: the glabellar complex or brow, lateral orbital region or "crow's-feet," the forehead, and "bunny lines." In the glabellar region, three chief muscles are treated, the procerus, the corrugator supercilii, and the accompanying depressor orbicularis oculi. The procerus, which is statistically larger in women, produces furrows at the base of the nose (radix). A powerful "wrinkler" of the nose, the procerus creates deep furrows with chronic activity. These can be treated with 3 to 5 units of BTX in one or two aliquots in the area of the radix (Fig. 1). Frequently, patients have limited procerus activity and do not require treatment to the muscle when primarily 


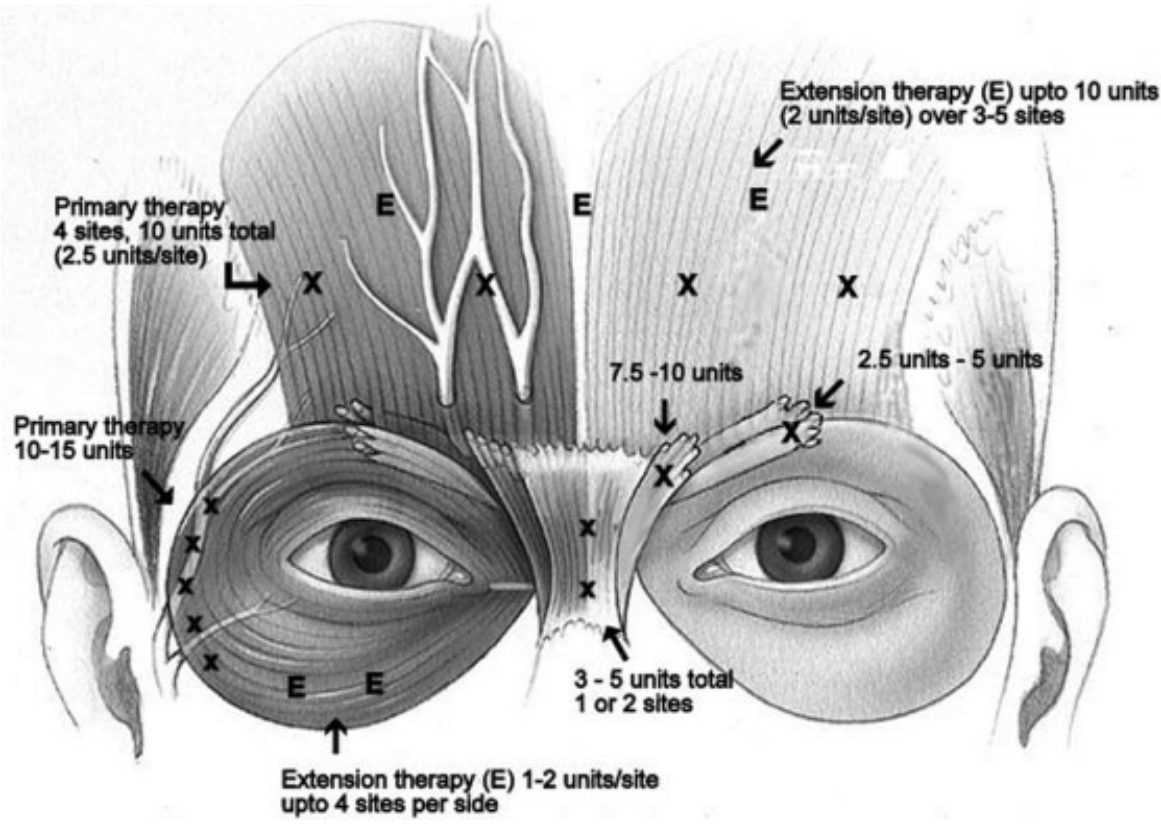

Figure 1 Anatomy of upper face demonstrating recommended injection sites and dosages for Botex cosmetics.

concerned with the vertical rhytides associated with the corrugators. Thus, treatment formulas that are universal are wasteful and unnecessary. $7,9,10,13,14$

The corrugator supercilii and depressor orbicularis oculi muscles are clinically indistinguishable, and their anatomy is poorly understood. In the vast majority of patients, the corrugator follows the course of the eyebrow and delicately interdigitates with the orbicularis oculi muscle laterally and frontalis muscle superiorly, which contrasts with schematic diagrams showing the tail (insertion) of the corrugator $1 \mathrm{~cm}$ above the brow. Effective treatment of the vertical glabellar rhytides requires that the bulk of the BTX dose be given into the corrugator at the clubhead of the eyebrow. A minimum of 7.5 units is injected into this area and 2.5 units in the scant muscle of the lateral brow to address the "recruitment" of this portion of the muscle and some of the horizontally orientated orbicularis. The second injection is directed at or slightly (within 1 or $2 \mathrm{~mm}$ ) above the level of the eyebrow (Fig. 1). With knowledge of the eyebrow complex muscles, it is understood that injections well above the midpupillary brow are of little value in targeting corrugator function and instead disable the lower portions of the frontalis muscle, possibly resulting in medial brow ptotis. $7,9,10,13,14$

The lateral orbital rhytides known as "crow's-feet" are treated with injection of an average of 10 units of BTX along a half-moon shape around the lateral aspect of the orbital rim. Injection begins just under the tail of the eyebrow and continues with three to four sites curving inferiorly and medially (Fig. 1). Ecchymosis can easily occur in this area, but size can be limited by applying pressure on evidence of a bleeding injection site. An important consideration in treating the lateral portion of the orbicularis oculi muscle is its affect on brow position. In its native state, the muscle is the most powerful depressor of the brow. The injection plane is immediately subcutaneous and $1 \mathrm{~cm}$ lateral to the rim to avoid diffusion into the orbit. Care is taken to not deeply inject inferiorly along the lower eyelid, as the upper portions of the zygomaticus muscle can be affecting, altering the smile. Neither has been a problem in the

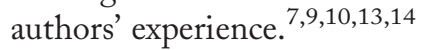

Horizontal rhytides of the forehead result from contraction of the underlying frontalis muscle, which originates from the galea aponeurotic layer superiorly and interdigitates with procerus, corrugator, and orbicularis oculi muscles over the brow region. The vertical pull of the muscle elevates the brow and retracts the scalp. Understanding the impact of frontalis muscle treatment on the brow position is a crucial part of the discussion with patients. It is important to inform them that complete effacement of lines will result in brow ptosis, which is usually not desired. Typical starting doses are 5 units per side in four to five 2.0- to 2.5unit aliquots evenly distributed across the forehead at least 1.5 to $2 \mathrm{~cm}$ above the brow (Fig. 1). It is important to include treatment of the lateral portion of the muscle to avoid the "Mr. Spock-like" deformity. Additional injections superior to this level can be used for further line effacement. The key to success in this area is modest, uniform dosing to avoid asymmetry and artificial appearance of the brow complex. Doses larger than 20 units are rarely warranted. $7,9,10,13,14$

"Bunny lines," which form radially over the nasal bones, can be treated by treating the underlying transverse nasalis muscle. The lines are created by contraction of the muscle, whose fibers course in a superomedial 
direction from its origin on the maxilla over the nasal bridge to fuse with its contralateral muscle and the aponeurosis of the procerus muscle. Treatment is targeted to the superior portion of the muscle with 2.5 units bilaterally. Inferiorly based injections can result in paresis of the levator labii superioris alaeque nasi and levator anguli oris manifesting as ipsilateral lip ptosis and an asymmetric smile. Conversely, overinjection superiorly can result in medial rectus paresis and epiphora. ${ }^{13}$

In the lower face, the primary targets for facial rejuvenation with BTX are the oral commissures and lip lines. Treating the depressor anguli oris (DAO) muscle results in the improvement of perioral appearance by softening the melomental folds, or "marionette lines," and downturned oral commissures, which relay sadness or disapproval. The DAO muscle arises from the oblique line of the mandible and inserts into the angle of the mouth, drawing it downward over time (Fig. 2). The muscle is injected directly with 3 units, $1 \mathrm{~cm}$ lateral and $1 \mathrm{~cm}$ inferior to the angle of the mouth, or where the nasolabial fold intersects the mandible. Caution is exercised to avoid injecting the lip depressors medially, which causes flattening of the contour of the lower lip when the patient attempts to form an "O," or injecting too high, which may alter speech and suction. ${ }^{7,9,15}$

The perioral lip lines are best treated with fillers and resurfacing, however the orbicularis oris muscle can be treated with BTX sparingly to reduce the repetitive pursing expressions that lead to the so-called smoker's lines. Generally, 4 to 6 units of BTX are placed with precise symmetry at the vermillion border superficially, in four to six 1-unit aliquots for the both the upper and lower lip. With larger doses, the risk of early problems including dysarthria and oral incompetence are increased. $7,9,15$

Aging of the neck manifests in vertical bands and horizontal necklace lines. Both of these problems are associated with the thin, broad-based platysma muscle.
The vertical bands occur as the platysma muscle separates in the midline with age and skin loses its elasticity, and the transverse lines result from pleating of the overlying skin perpendicular to the underlying muscle. Ten units of BTX are typically injected into the central third of vertical bands in two to four sites to re-create the cervicomental angle (Fig. 3). Care is taken to stay within the belly of the muscle and not deep into the strap muscles. The horizontal necklace lines are treated with subcutaneous injections of 15 to 20 units just above and below the lines. Patient selection is very important in treating the aging neck with neuromodulators, as injection does not correct skin laxity or fatty deposits. ${ }^{7,9,13-15}$

\section{FILLERS}

\section{Biologic Fillers}

The first successful commercially available injectable filler was bovine collagen, approved by the FDA in 1981. Commercial products included Zyderm and Zyplast (Inamed Corp., Santa Barbara, CA). ${ }^{16}$ These products are no longer used partly because of the availability of alternative products that are more effective and easier to use. Disadvantages to the use of these products includes the need for refrigeration, short duration of action (usually 3 months), and need for allergy testing. ${ }^{7}$ Also, good initial results could be altered by delayed hypersensitivity reactions.

Evolence (Colbar Life Science Ltd., Herzliya, Israel) emerged in the soft tissue filler market in mid-2008. The product contains purified porcine collagen at a concentration of $35 \mathrm{mg} / \mathrm{mL}$. Despite being a collagen product, it does not require skin testing because of the enzymatic digestion during processing, which removes the antigenic portion of the molecule. The extensive cross-linking of the collagen with a naturally

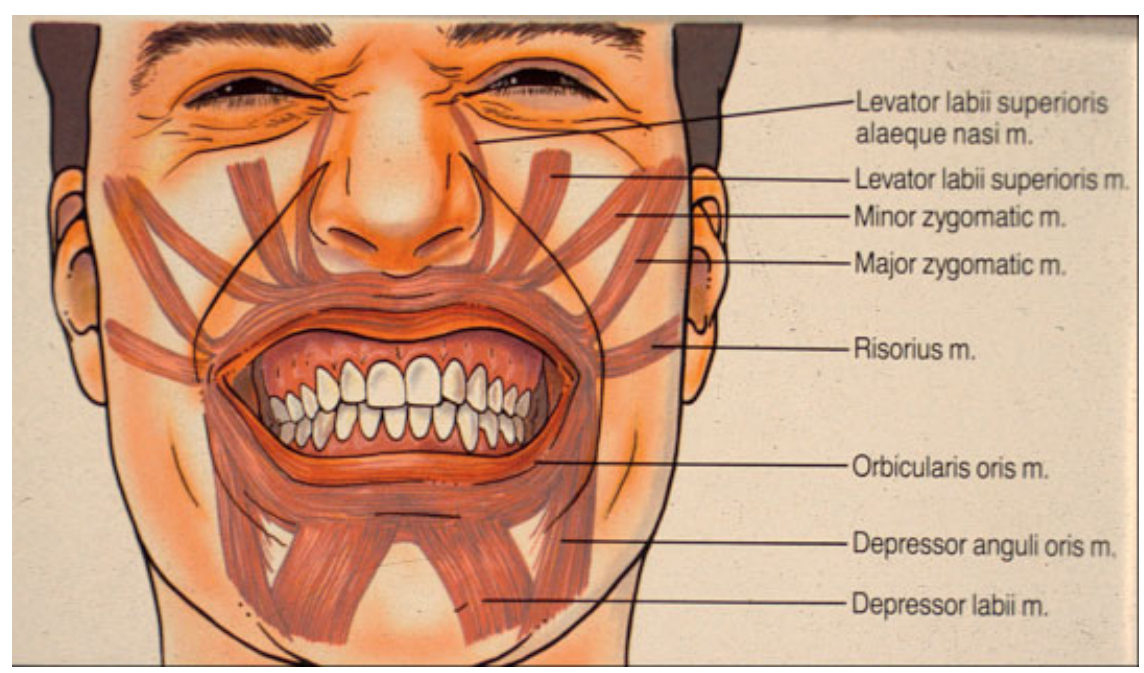

Figure 2 Lower facial musculature pertinent to treatment of the marionette lines. 


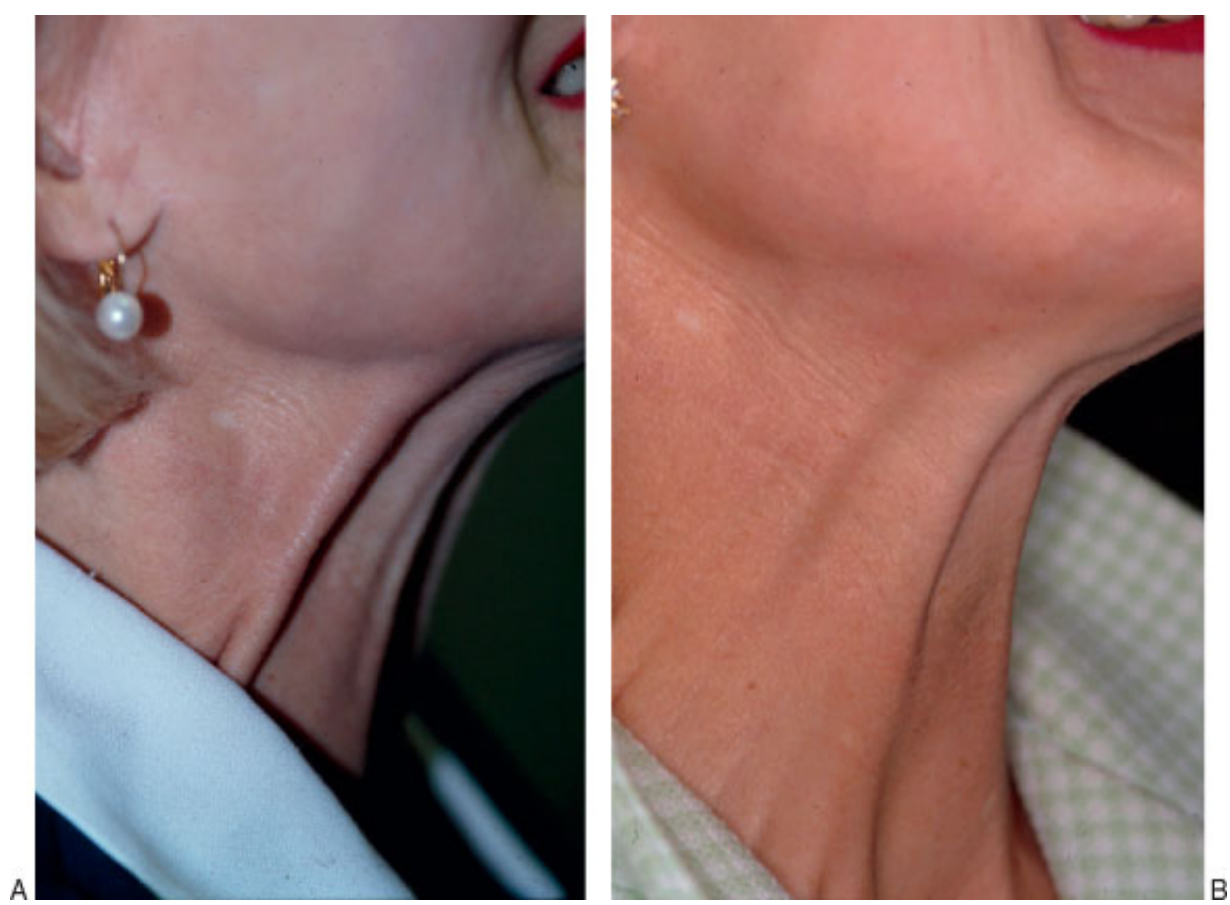

Figure 3 (A) Before and (B) after photos of platysmal bands treated with Botox injections.

occurring sugar metabolite via Glymatrix technology extends the duration of the product in vivo to at least 12 months. ${ }^{1,2}$ Evolence is being marketed by Johnson and Johnson, who acquired the product from Colbar, Inc., as the "event filler" for deep lines and wrinkles. The hemostatic properties of collagen almost completely eliminate bruising and swelling and allow patients to engage in social activities with virtually no downtime. Other advantages of the product include a softer and more natural feel compared with that of products with equally long duration of correction. The product's current formulation requires subdermal injection as injection too superficially can result in milia. Another formulation, Evolence Breeze, may soon become available for injection into fine lines and for lip augmentation.

Cosmoderm and Cosmoplast (Allergan Inc.) are human dermis-based collagen fillers $(35 \mathrm{mg} / \mathrm{mL})$ designed for effacing fine lines, skin defects, and scars as well augmenting the lip border. ${ }^{16}$ The products are formulated with $0.3 \%$ lidocaine to assist with anesthesia during injection and do not require allergy testing. Unfortunately, Allergan recently announced that they would scale down the manufacturing of the product and may only be able to supply the products through $2010 .{ }^{17}$ This is probably secondary to economic factors and not the effectiveness of the products.

Hylaform (Biomatrix, Inc., Ridgefield, NJ) is a HA filler derived from rooster combs. The product has a high degree of cross-linking and a HA concentration of $5.5 \mathrm{mg} / \mathrm{mL}$. Hylaform is injected into the subdermal plane and used for deeper lines and folds. Clinical phase III studies revealed that Hylaform had at least equal results to those of Zyplast for correction of nasolabial folds for at least 4 months. ${ }^{18}$ Other HA fillers currently available are manufactured from non-animal sources, chiefly Streptococcus fermentation.

\section{Manufactured Fillers}

\section{HYALURONIC ACIDS}

The currently available non-animal source manufactured HA fillers in the United States are Restylane, Perlane, Juvederm, and Prevelle and Prevelle Silk. Hyaluronic acid is a naturally occurring substance in the human body and serves as the ground substance of dermis, fascia, and other tissues. There are several advantages in using the HA fillers including the immediate reversibility with hyaluronidase and the lack of antigenic specificity decreasing the risk of allergic response. Another property that makes these products useful clinically is the hydrophilic nature, allowing $1 \mathrm{~g} \mathrm{HA}$ to bind up to $6 \mathrm{~L}$ water.

Restylane (Medicis, Scottsdale, AZ) was introduced in 1996 as the first non-animal stabilized HA. Derived from the fermentation of Streptococcus species, the molecules are then cross-linked by the addition of 1,4-butanediol diglycidyl ether to increase surface area and slow down the natural breakdown of the product. The concentration of HA in Restylane is $20 \mathrm{mg} / \mathrm{mL}$, and the particles average $400 \mathrm{um}$. Manufactured identically, Perlane (Medicis) is a thicker product as a result of larger particle size, containing only 8000 gel beads per milliliter, whereas Restylane contains an average of 100,000 gel beads per milliliter. ${ }^{19}$ The decreased surface area of 
Perlane theoretically extends its duration of correction compared with the 6 months expected with Restylane. One downside of Perlane is that because of its larger particle size, it requires injection through a 27 -gauge needle, increasing patient discomfort, compared with the 30-gauge needle for Restylane. Perlane is designed for injection into deep folds and for facial augmentation, whereas Restylane can be used for these applications and more superficially.

Juvederm (Allergan Inc.) has a HA concentration of $24 \mathrm{mg} / \mathrm{mL}$. Two configurations of Juvederm are currently available: Juvederm Ultra and Juvederm Ultra Plus. Juvederm Ultra is less dense and more appropriate for medium-depth wrinkle correction, such as nasolabial folds, lip augmentation, forehead wrinkles, glabellar lines, obvious mild to moderate nasal furrows, and cheek wrinkles. Juvederm Ultra Plus is more extensively crosslinked, resulting in a more robust product for deeper, more pronounced facial folds and for restoring volume to the cheeks and lips. A recent development is the packaging of Juvederm with a customized 27-gauge needle with a smaller outer diameter that allows for better patient comfort without alteration of product flow. Juvederm sustains clinical correction for at least 6 months in dynamic areas of the face. It may last up to a year or more in adynamic areas, which is reflected by it FDA approval.

Prevelle and Prevelle Silk (Mentor Corp., Irving, TX) are also HA products; however, they are designed to be injected superficially into the dermis to allow for fine lines of the face. The expected duration of these products is 3 to 4 months. Prevelle Silk differs from Prevelle in that it contains $0.3 \%$ lidocaine, which obviates the need for additional local anesthetic when filling the fine lines in multiple areas of the face, which can be difficult to anesthetize with local nerve blocks. With the recently announced future discontinuation of the Cosmoderm product, the Prevelle products will be the primary filler for fine lines. Other filler product lines including Evolence are not far from launching materials that will compete in the fine line market.

Belotero (Merz Pharma, Franfurt, Germany), a monophasic double cross-linked HA filler available in Europe, has completed phase III FDA trials for subdermal filling. Merz developed CPM (Cohesive Polydensified Matrix) production technology setting Belotero apart from conventional HA fillers, which are biphasic. The unique structure of the stable matrix completely fills the intradermal space and hence leads to a lasting and natural cosmetic result with soft dermal transitions. ${ }^{20}$

\section{NON-HYALURONIC ACID FILLERS}

Radiesse (Bioform, Inc. Franksville, WI) is composed of microscopic calcium hydroxyapatite particles (ranging in size from 25 to $40 \mu \mathrm{m}$ ) suspended in a carboxymethyl- cellulose gel. Radiesse allows for volume restoration and acts as a framework for fibroblastic in-growth in soft tissue. Histologic studies reveal that the microspheres disappear at 9 months, but clinically for facial applications the duration is in the range of 6 to 12 months depending on the injection site and technique. ${ }^{21}$ The use of Radiesse is limited to deeper folds and facial augmentation placing the product in the subdermal level resulting in natural appearance and feel.

\section{Polymers/Particles}

Silicone, a polymer of dimethylpolysiloxane, sold as Silikon 1000 (Alcon Laboratory, Inc., Fort Worth, TX), may be used off-label for subdermal filling. The product is injected with a 30 -gauge needle in microdroplets of 0.01 $\mathrm{mL}$. Each microdroplet produces augmentation by formation of a fibrous capsule around each droplet of silicone, which can take weeks. While injecting silicone, one undercorrection should be practiced because of the permanent effects, and subsequent treatments should be at 6-week or greater intervals. Numerous complications, including inflammation, induration, discoloration, ulceration, migration, and granuloma formation, have limited its use. Some believe these complications are caused by improper injection technique, injecting too much silicone, or the quality of the silicone. ${ }^{22}$

Polymethylmethacrylate (PMMA) microspheres are available as Artefill (Artes Medical Inc., San Diego, CA), a suspension of 30- to $40-\mu \mathrm{m}$ diameter spheres in $3.5 \%$ bovine collagen. The product's immediate effect is correction with the collagen and then permanent replacement with new collagen as a result of the microgranuloma formation induced by PMMA. Artefill is designed for subdermal injection, and serial treatment is recommended by the manufacturer to ensure a gradual buildup of the product and to avoid overinjection and/or a granulomatous and fibrous tissue response.

The permanent fillers can be problematic because of the tissue reactions they produce as well as the irreversibility of their filling effects, which result in cosmetic morbidity, requiring wide local excision for treatment. Irregularity and nodularity are the common complaints. For these reasons, these products are not used in our practice for facial rejuvenation. Nonetheless, as long as the patient and physician understand these risks, both silicone and Artefill can be effective and socalled permanent treatments. However, it would be interesting to follow patients injected with permanent fillers to see if the correction generated holds up as tissue becomes deflated and gravity continues to act over time.

\section{APPLICATIONS OF FILLERS}

Fillers can be used in all levels of the face for rejuvenation and correction of anatomic deficiencies. In the upper 
face, the most frequently treated problems are the fine lines of the forehead and the tear-trough deformity of the lower eyelid. The horizontal and vertical lines of the forehead and brow respectively are best relaxed with the use of BTX. However, lines that are recalcitrant to this treatment can be treated with soft tissue fillers. The best choice for fine lines is Cosmoderm or Cosmoplast, but as the supply diminishes, Prevelle Silk will be the product of choice. Injection is intradermal, using a serial puncture technique. For deeper furrows, HA products such as Juvederm or Restylane could be used subdermally using a linear threading technique. Radiesse could be used effectively as well.

Achieving good results in the tear trough or nasojugal fold is very challenging. However, a good result is very gratifying as full correction can last as long as 2 to 3 years, probably as a result of the relative immobility in this area (Fig. 4). The authors' product preference is Juvederm or Restylane. However, the choice of product is only a small part of success in this area, the most important factor being technique. Topical anesthetic creams and ice only are used as injection of filler is well tolerated in this area. Injection of local anesthetic would distort the contours of these delicate tissues and is discouraged. The patient should be seated upright, and gaze should be fixed on the horizon to allow for deliberate injection into the deficient areas, as fat pads can be reoriented by upward and downward gaze and of course by gravity. The product is injected along the orbital rim staying preseptal to avoid exacerbating the pseudoherniation of orbital fat that causes the deformity. Ecchymosis occurs in 1 in 5 patients in this area because of the thin skin and the needle passing through the orbicularis muscle. ${ }^{23}$ If bruising becomes evident, the injection should be halted, and pressure should be applied to the area for several minutes to minimize the effects. After injection is complete, the area should be massaged to ensure that product is evenly distributed. Edema sets in quickly, and the patient should be reassessed to decide if additional correction is needed or partial dissolution of the product is required with the use of hyaluronidase. This should be at a minimum of 4 weeks later to allow for the water bound by HA to dissociate. A well-known complication of HA injection in this area, although not the only complication, is the Tindel effect, or bluish appearance of the product under the very thin skin. This problem can also be corrected with hyaluronidase. Other potential complications include arterial occlusion or embolization, which could result in blindness. Fortunately this is theoretical but nevertheless should be discussed with patients when obtaining informed consent.

In the lower face, the primary areas of soft tissue filler include the nasolabial folds, the lips, and the oral commissures. For the nasolabial folds, a wide variety of products can be used from collagens to HA products and manufactured non-HA products. Also, the products can be injected superficially into the dermis to efface fine lines or deeper into the subdermal level to smooth the appearance of the nasolabial fold itself. The authors' technique includes providing a block of the infraorbital nerve with local anesthetic, although topical anesthetic and ice may also provide adequate patient comfort. The preferred products for the deep nasolabial folds are Evolence and Radiesse because they provide a long duration of correction. However, in the first-time filler

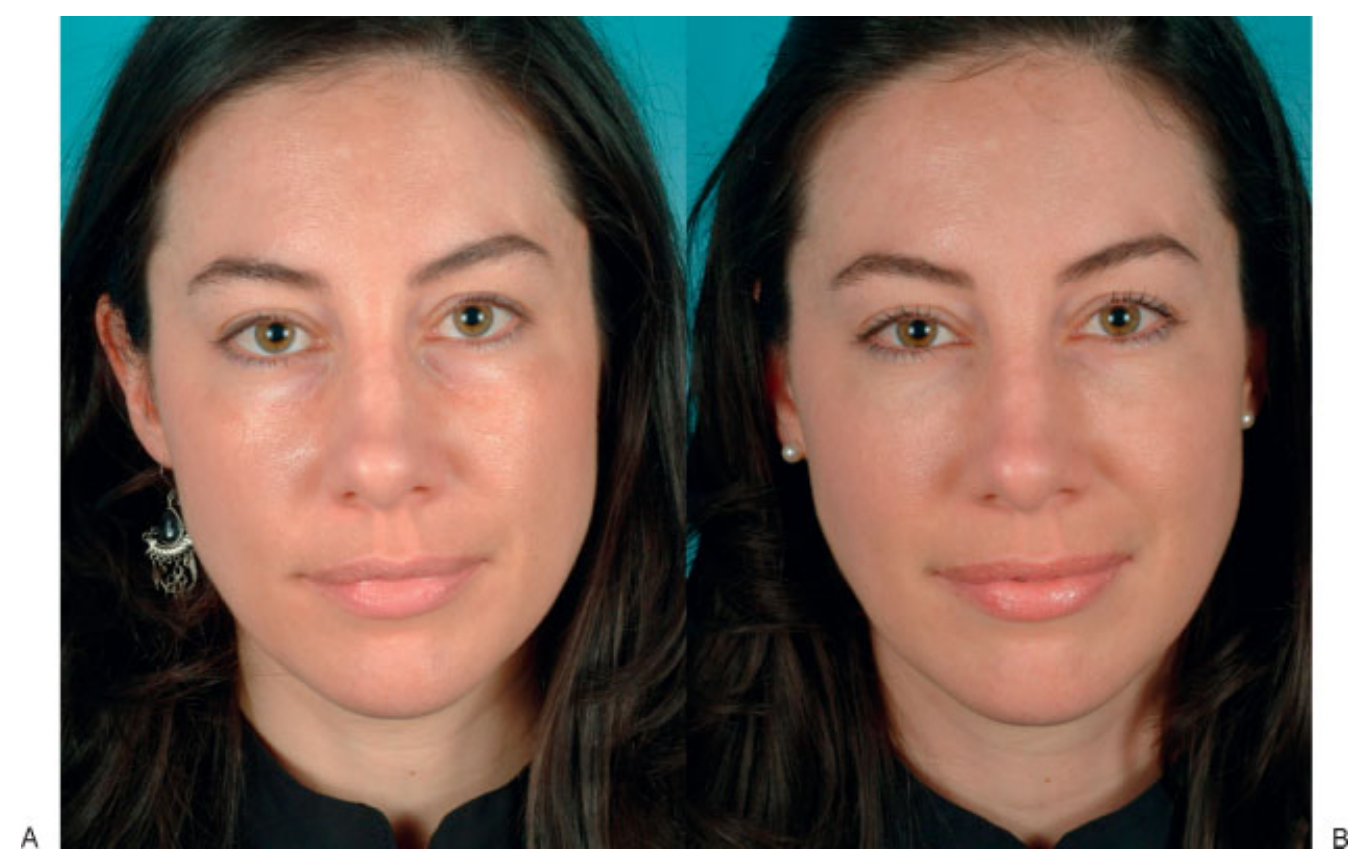

Figure 4 (A) Before and (B) after photos of Restylane injection into nasal jugal groove area. 
A
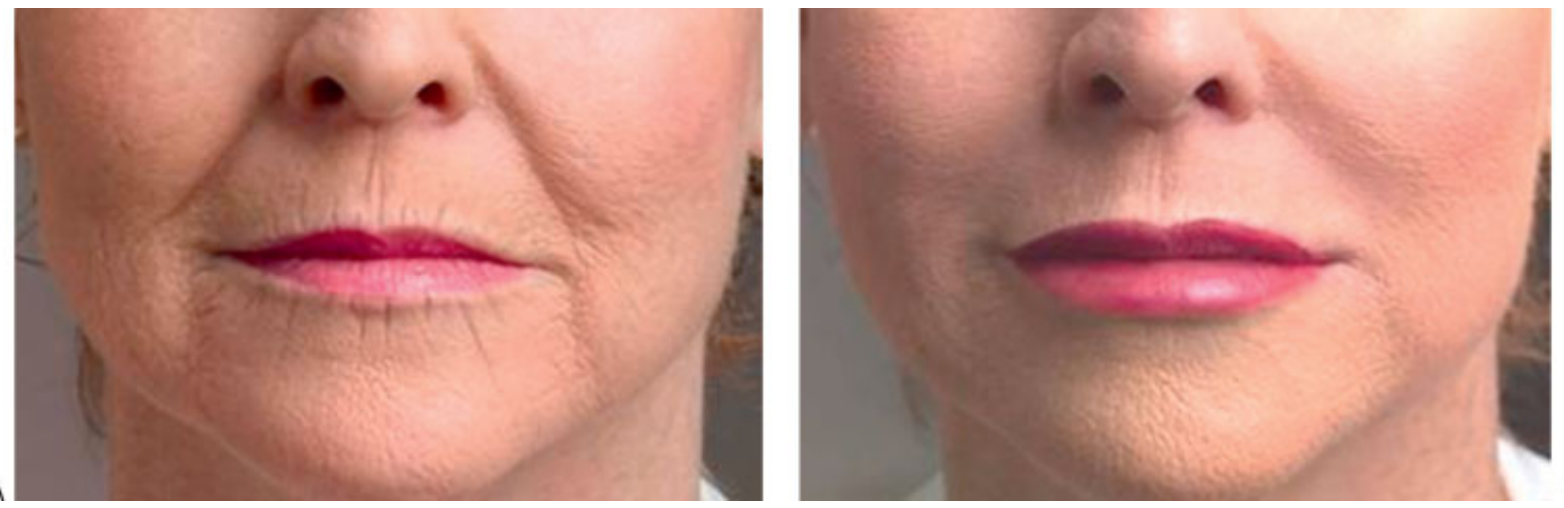

B

Figure 5 (A) Before and (B) after photos of nasolabial folds injected with Restylane.

patient, it may be advisable to use a reversible HA product such as Restylane, Perlane, or Juvederm (Fig. 5). When injecting the nasolabial fold, a linear threading technique is used, placing the product in a retrograde fashion. For successful rejuvenation of this area, the triangle formed at the superior aspect of the fold by the junction of the nasal ala and cheek must be filled. It important to stop injecting before the dermis is encountered on withdrawing the needle to prevent possible nodularity or Tindel effect by being too superficial. With the HA products, some overfilling is suggested, whereas minimal overcorrection is suggested with Radiesse and none for Evolence. To address the fine etched lines sometimes associated with the nasolabial fold, Prevelle and Prevelle Silk are effectively injected using a serial puncture technique. The common complications to injection in this area include the usual risks of soft tissue fillers such as local inflammation, ecchymosis, and edema.

Both the upper and lower lips are a common target for rejuvenation and augmentation. The aging lip appears thin as the orbicularis oris muscle loses bulk, the Cupid's bow flattens, and the white portion of the lip lengthens. Patients frequently complain of not being able to apply cosmetics without running of the products. Both the vermillion border and body of the lip can be treated. The authors' product of choice is Restylane in this area because of the relative firmness of the product and its ability to contour the lip, although Cosmoderm or Prevelle could be used along the lip border as well (Fig. 6). Injection technique includes the use of topical anesthetic and ice as well as nerve blocks frequently. Antiviral prophylaxis is given to patients with a history of cold sores or herpes simplex. ${ }^{24}$ The vermillion border is enhanced by injection into the potential space between the red and white lip. Frequently, this space can be entered with the needle, and product can be injected along the entire length of the lip by hydrodissection, providing uniform placement of the product. Alternatively, a serial puncture technique can be used. The body of the lip is augmented by submucosal injection with focus on the central one-third of the lower lip. ${ }^{23}$ Further plumping and eversion can be achieved by everting the lip and placing several linear "beads" caudal to the wet lip border.

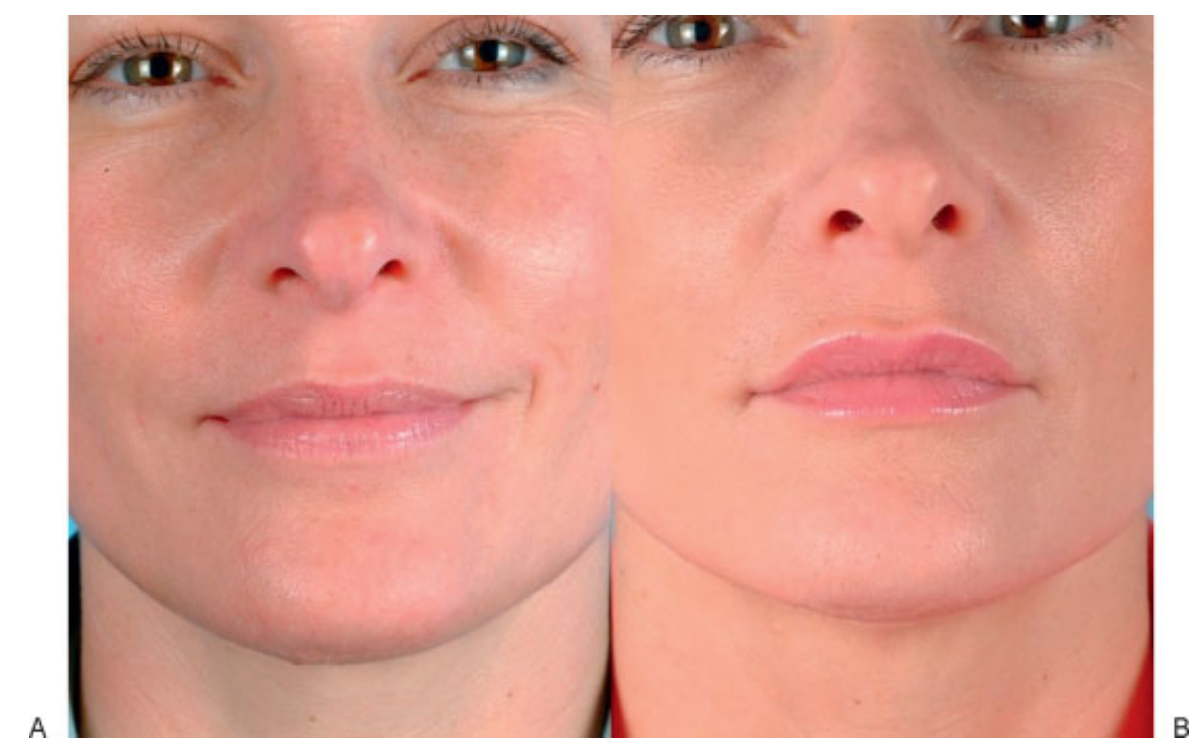

Figure 6 (A) Before and (B) after photos of microcheilia treated with Restylane injection. 


\section{Injection techniques}

Serial puncture

technique
Linear threading technique

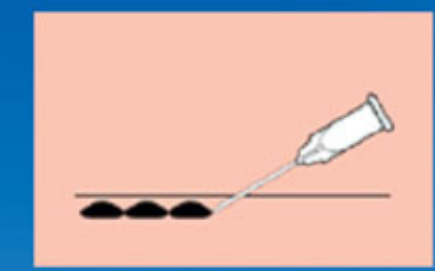

Fan technique

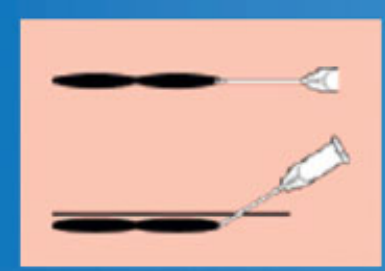

Cross-hatching technique
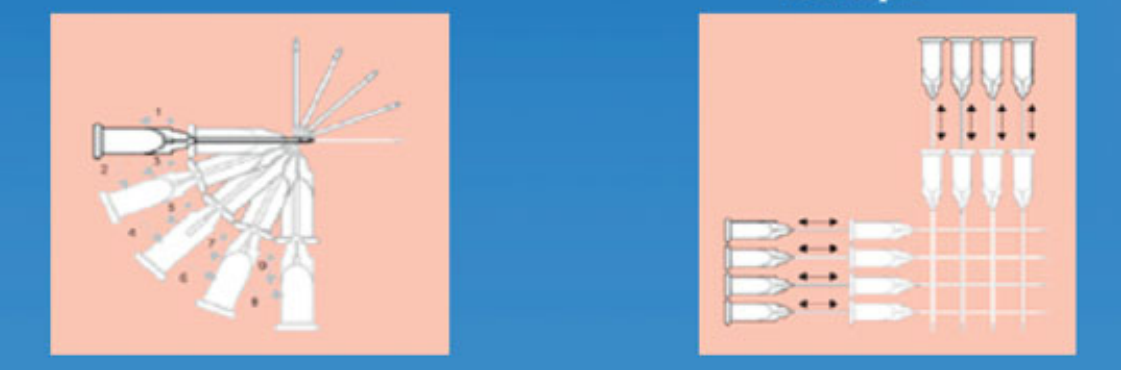

Figure 7 Various injection techniques.

The deep marionette lines and downturned oral commissures can be reversed by the injection of one among a variety of products. Any one of the dermal fillers can be injected into this area, and choice of product is often dictated by the use of product in other areas of the face, lessening "waste" of filler. The authors' choice of deep filler in this area is a HA or collagen product versus Radiesse, which may be too firm for this area. For the fine lines in this area, as in others, Cosmoderm or Prevelle are used. Injection preparation for the oral commissures is similar to that for other areas of the face, using topical anesthetic, ice, and local nerve blocks with care, as in other areas, and not using large volumes of local anesthetic and distorting the area to be filled. The fillers are placed in the subdermal plane in a triangle formed with its base at the lower lip.

Aging of the face is characterized by formation of rhytides by repetitive actions of facial muscles, elastosis of skin from sun damage, and the loss of subcutaneous fat. The loss of volume in the face can be treated with subdermal fillers as well. The primary areas of concern are the cheeks, along the zygoma, the prejowl sulcus, and temples. Volume enhancement in these areas is achieved with injection subdermally or deeper with a long-lasting filler such as Radiesse, although the softer HA products could also be used. The injection technique should include fanning and cross-hatching of product for uniform filling, without perceptible irregularities (Fig. 7). Patient comfort is achieved with combinations of topical and local anesthetic. Complications of volume enhancement in these areas are similar to those of filler injections in other areas of the face.

\section{CONCLUSION}

Minimally invasive facial rejuvenation has continued to evolve over the past several years by way of the increasing development of new soft tissue fillers and different formulations of neuromodulators. It is crucial to understand the muscular anatomy and changes associated with aging of the face to effectively treat patients. Just as important is proper selection of products for patient goals, and well thought out and careful technique to optimize results and minimize complications. Perhaps the most critical key to success with injectables is proper patient selection and counseling. The limitations and indications of injectable treatments must be understood by the physician and patient to ensure the greatest possible satisfaction with minimally invasive facial rejuvenation.

\section{REFERENCES}

1. Narins RS, Brandt FS, Lorenc ZP, et al. A randomized, multicenter study of the safety and efficacy of Dermicol-P35 and non-animal-stabilized hyaluronic acid gel for the correction of nasolabial folds. Dermatol Surg 2007;33(Suppl 2): S213-S221; discussion S221 
2. Narins RS, Brandt FS, Lorenc ZP, Maas CS, Monheit GD, Smith SR. Twelve-month persistency of a novel ribose-crosslinked collagen dermal filler. Dermatol Surg 2008;34(Suppl 1): S31-S39

3. Eppley BL, Dadvand B. Injectable soft-tissue fillers: clinical overview. Plast Reconstr Surg 2006;118:98e-106e

4. Reisman NR. Ethics, legal issues, and consent for fillers. Clin Plast Surg 2006;33:505-510

5. Ali MJ, Ende K, Maas CS. Perioral rejuvenation and lip augmentation. Facial Plast Surg Clin North Am 2007;15: 491-500

6. Engelman DE, Bloom B, Goldberg DJ. Dermal fillers: complications and informed consent. J Cosmet Laser Ther 2005;7:29-32

7. Maas CS, Yu KC, Egan KK. Neuromodulators and injectable soft tissue substitutes. In: Papel ID, Frodel JL, Holt GH, et al, eds. Facial Plastic and Reconstructive Surgery. 3rd ed. New York, NY: Thieme Medical Publishers; 2009:337353

8. Maas CS, Ende KH, Lewis DA. Soft tissue fillers. In: Truswell W, ed. Surgical Facial Rejuvenation: A Roadmap to Safe and Reliable Outcomes. New York, NY: Thieme Medical Publishers; 2008:131-138

9. Petrus GM, Lewis D, Maas CS. Anatomic considerations for treatment with botulinum toxin. Facial Plast Surg Clin North Am 2007;15:1-9, v

10. Maas CS. Botulinum neurotoxins and injectable fillers: minimally invasive management of the aging upper face. Facial Plast Surg Clin North Am 2006;14:241-245

11. Ramirez AL, Reeck J, Maas CS. Botulinum toxin type B (MyoBloc) in the management of hyperkinetic facial lines. Otolaryngol Head Neck Surg 2002;126:459-467

12. Lowe PL, Patnaik R, Lowe NJ. A comparison of two botulinum type a toxin preparations for the treatment of glabellar lines: double-blind, randomized, pilot study. Dermatol Surg 2005;31:1651-1654

13. Loos BM, Maas CS. Relevant anatomy for botulinum toxin facial rejuvenation. Facial Plast Surg Clin North Am 2003; 11:439-443

14. Stupak HD, Maas CS. New procedures in facial plastic surgery using botulinum toxin A. Facial Plast Surg Clin North Am 2003;11:515-520

15. Dayan SH, Maas CS. Botulinum toxins for facial wrinkles: beyond glabellar lines. Facial Plast Surg Clin North Am 2007;15:41-49, vi

16. Wise JB, Greco T. Injectable treatments for the aging face. Facial Plast Surg 2006;22:140-146

17. Allergan, Inc. $10-\mathrm{k}$ sec filing. Available at: http://sec.edgaronline.com/allergan-inc/10-k-annual-report/2007/03/01/Sec tion3.aspx. Accessed May 15, 2009

18. Monheit GD. Hyaluronic acid fillers: Hylaform and Captique. Facial Plast Surg Clin North Am 2007;15:77-84, vii

19. Brandt FS, Cazzaniga A. Hyaluronic acid fillers: Restylane and Perlane. Facial Plast Surg Clin North Am 2007;15: 63-76, vii

20. Merzpharmaceuticals. Company press release 2005. Available at: http://www.merz.com/press/press-releases/company/2005/ 05/12/01/. Accessed May 15, 2009

21. Ahn MS. Calcium hydroxylapatite: Radiesse. Facial Plast Surg Clin North Am 2007;15:85-90, vii

22. Narins RS, Beer K. Liquid injectable silicone: a review of its history, immunology, technical considerations, complications, and potential. Plast Reconstr Surg 2006;118(3 Suppl): $77 \mathrm{~S}-84 \mathrm{~S}$

23. Finn JC, Cox S. Fillers in the periorbital complex. Facial Plast Surg Clin North Am 2007;15:123-132, viii

24. Kelly PE. Injectable success: from fillers to Botox. Facial Plast Surg 2007;23:7-18; discussion 19-20 